and Belgium, in: International Labour Review, 133, 5-6.

Zeitlin, J. (1991): 'Reconfiguration of the Market and the Use of Computerised Technology' in Mitter, S. (Ed.), (1991) Computer-aided Manufacturing and Women's Employment, Springer-Verlag, London.

》

\section{"Standortdebatte" und "Neue Armut": Bundesdeutsche Illu- strationen zum EU-Projekt SOWING}

\author{
von Günther Frederichs, ITAS
}

\section{Gibt es nationale Sonderwege in die "Informationsgesellschaft"?}

Das EU-Projekt SOWING spannt den Bogen seiner Themen von den Kommunikations- und Informationstechnologien über die technologische Praxis von Wirtschaftsunternehmen bis hin zu neuen Formen sozialer Ausgrenzung. Damit wird von vornherein eine auf die technologische Entwicklung verengte Perspektive vermieden und es werden die Wechselwirkungen mit anderen gesellschaftlichen Vorgängen in die Beobachtung mit einbezogen. Überraschenderweise kann die "Informationsgesellschaft" auch bei dieser komplexeren Betrachtungsweise immer noch unabhängig von den nationalen Besonderheiten eines Landes beschrieben werden, zumindest in erster Annäherung. Die Ausgangsbedingungen sind diejenigen, die generell in den Industrienationen am Ende des 20. Jahrhunderts vorzufinden sind - ein bemerkenswerter Aspekt der These vom Entstehen einer globalen Gesellschaft, wie es die Theoretiker der Moderne sehen!

Wenn es jedoch darum geht, die Hypothesen und Prognosen empirisch zu verifizieren, und wenn ein Vergleich der technologischen Praxis in verschiedenen europäischen Regionen vorgenommen werden soll, dann rücken die jeweiligen Kontexte als "intervenierende Variable" ins Bild. $\mathrm{Zu}$ fragen ist dann, aus welchen nationalen Erfahrungen sich die Diskussion speist und welches die Entwicklungen eines
Landes sind, deren Extrapolationen und Verallgemeinerungen in die Prognose der Informationsgesellschaft einmünden. Wenn es nationale Sonderwege in die Informationsgesellschaft gibt, dann sind es spezifische Konstellationen der jeweiligen Länder, die der Entwicklung eine spezielle Ausprägung geben. Angesichts der vielbeschworenen Vielfalt der Länder und Kulturen Europas sollte man unterschiedliche $\mathrm{Mu}$ ster der Entwicklung erwarten.

Welche Kontexte in die Beobachtung einbezogen werden, ergibt sich aus den zentralen Themen von SOWING: die technologische Praxis von Wirtschaftsunternehmen und die Entstehung neuer Formen sozialer Ausgrenzung am Arbeitsmarkt. Sie lenken den Blick auf das unternehmerische Handeln im Rahmen des jeweils geltenden Produktions- und Regelungsmodells (Czada 1998). Durch die Verwendung des lebensweltlichen Begriffs der "Praxis" öffnet sich der Blick den vielfältigen demographischen, infrastrukturellen und kulturellen Einflüssen. Es ist nicht zuletzt das System der industriellen Beziehungen, in dem die Konfliktkultur eines Landes zum Ausdruck kommt und so der technologischen Praxis einer Firma eine nationale Färbung gibt.

\section{Das "Modell Deutschland" und die Standortdebatte}

Die wohlfahrtsstaatliche Einbettung der westdeutschen Marktwirtschaft besteht traditionell in "verbandlicher Selbststeuerung": Gewerkschaften, Arbeitgeberverbände und Industrievereinigungen regulieren wichtige Bereiche autonom oder im Austausch mit staatlichen Instanzen. Diese nach dem Konsensprinzip funktionierende Konfiguration konnte bis zur Wiedervereinigung die wichtigsten Voraussetzungen des einst international gepriesenen "Modells Deutschland" erfolgreich realisieren: die Wettbewerbswirtschaft, die sektorale Selbstorganisation, die Strukturpolitik auf Länderebene, die Tarifautonomie und last not least das Wirtschaftswachstum (Czada 1998). Die wesentlichen Merkmale sind

- die Einbindung von Gewerkschaften und Wirtschaftverbänden in die allgemeine Wirtschaftspolitik ("Konzertierte Aktionen", "Bündnis für Arbeit") 
- die kooperative Konfliktbewältigung durch das Tarifvertragswesen (Tarifautonomie, Flächentarifvertrag und Lohnführerschaft einzelner Tarifbezirke)

- die bankenzentrierte Industriefinanzierung einschließlich weitreichender Überkreuzverflechtungen von Aufsichtsratsmandaten.

Ergänzend wird vom "Produktionsmodell Deutschland" gesprochen, wobei es um die "einzigartige Konfiguration von institutionellen Arrangements zur Gestaltung und Steuerung der Produktion" geht (Czada 1998). Hierzu gehören

- die betriebliche und überbetriebliche Mitbestimmung (Müller-Jentsch 1995)

- das duale System der Berufsausbildung (Beicht et al. 1997)

- das "Meistersystem" in der Produktionsorganisation (Behrens et al. 1996).

Die internationale Aufmerksamkeit richtet sich vor allem auf den speziellen Gegensatz dieses Modells zum "anglo-amerikanischen Modell": Der Wohlfahrtsmechanismus des Staates sorgt für ein vergleichsweise eng gewobenes soziales Netz von Renten, Bildung und Gesundheitsversorgung, ähnlich wie es auch in Frankreich und den Niederlanden ("Rheinmodell") der Fall ist. Diese Sicherung bestimmter Verpflichtungen der Wirtschaft gegenüber dem Gemeinwesen ist in England und den USA weniger gegeben. Dort wird die Unterordnung der staatlichen Bürokratie unter die Wirtschaft betont, verbunden mit einer höheren Bereitschaft, das vom Staat geschaffene Sicherheitsnetz zu lockern (Sennett 1998: 66; siehe aber zum vergleichsweise "geringen Niveau normativer Konflikte" in Deutschland Kaufmann 1997).

Seit Ende der achtziger Jahre unterliegt das "Modell Deutschland" zunehmend der Kritik. Politiker, Gewerkschafter und Wissenschaftler beklagen, daß die Erfolgskonzepte, die das "Modell Deutschland" einst auszeichneten, neuen Anforderungen nicht mehr gewachsen seien. Dies sind einerseits die aus der Diskussion um die "Informationsgesellschaft" bekannten Fragen der internationalen Wettbewerbsfähigkeit und des sektoralen Strukturwandels, die insofern kein deutsches Spezifikum darstellen. Daneben gibt es aber die besondere Belastung durch die deutsche Vereinigung, ein Faktor, der nach Darstellung von Czada (1998) mindestens gleichgewichtig neben den zuerst genannten Herausforderungen des deutschen Wohlfahrtsstaates stehe und dennoch auf nicht ganz nachvollziehbare Weise in der deutschen Öffentlichkeit kein Thema sei.

In der "Standortdebatte" wird in neoliberaler Ausrichtung angesichts veränderter weltwirtschaftlicher Rahmenbedingungen eine ungünstige Position Deutschlands im globalen Wettbewerb formuliert. Die Gründe dafür werden in den Arbeitskosten, Arbeitszeiten, Betriebszeiten und in der Unternehmensbesteuerung gesehen. Die Unternehmen würden darauf verstärkt mit der Auslagerung von Produktion ins kostengünstigere Ausland reagieren (Kroker 1995). Diese Debatte folgert (verbunden mit der Warnung vor Arbeitsplatzverlusten) als politische Empfehlung die Deregulierung bisheriger Entwicklungen deutscher Arbeitsmarkt- und Steuerpolitik.

Natürlich gibt es hierzu Differenzierungen. So deuten zum Beispiel die empirischen Daten an, daß die Produktionsverlagerungen deutscher Unternehmen primär innerhalb Europas bleiben (Wortmann, Dörrenbächer 1997), also im gemeinsamen Wirtschaftsraum. Ferner seien diese Auswirkungen auf den deutschen Arbeitsmarkt gering, da kaum Arbeitsplätze ins Ausland verlagert würden. Die Expansion der Unternehmen vollziehe sich im Ausland vorrangig durch Übernahmen und weniger durch Auslagerung (a.a.O.). Es wird auch darauf hingewiesen, daß man die Standortdebatte nicht auf Faktoren wie Arbeitskosten und Auslandsinvestitionen reduzieren dürfe. Berücksichtigt werden müßten auch die Arbeitsproduktivität, das Arbeits- und Tarifrecht, die Infrastruktur, die Qualifikation der Arbeitnehmer wie der Unternehmer und Manager, das Niveau von Forschung und Wissenschaft, die Belastung mit Steuern und Abgaben und der soziale Frieden. Die Zukunft des Standortes Deutschland hänge nicht allein an der Wirtschaft, sondern Human-Kapital und Lebensqualität würden bei vielen Standortentscheidungen den Ausschlag geben (Döring et al. 1995; Jagoda 1996). Es ist dies eine Forderung, die das SOWING-Projekt sicherlich mit dem Begriff der "technologischen Praxis" für sich in Anspruch nehmen kann. 
Aber gerade auf diese vielfältigen Faktoren, die das einst gepriesene "Modell Deutschland" ausmachten, richtet sich die Sorge einer ganz "anderen Standortdebatte" (SOFI 1995). Der Hintergrund ist die hohe Arbeitslosigkeit in Deutschland und die Befürchtung, daß sich ihre strukturellen Ursachen der politischen Steuerung entziehen. Die Aussage, daß die Bundesrepublik nicht nur im Osten, sondern auch im Westen einen Prozeß tiefgreifender Umwälzungen erfährt, wird zunehmend zum Bestandteil gegenwärtiger Krisendiagnosen. Das "Modell Deutschland" sei nur noch in Umrissen erkennbar (Czada 1998).

\section{3. "Soziale Ausgrenzung": neue Dimen- sionen der Sozialen Frage}

Der Begriff der „Social Exclusion“, also der Ausgrenzung von Menschen aus der Gesellschaft, zum Beispiel psychisch Kranken, Behinderten und Armen, neuerdings aber auch zum Beispiel alleinerziehenden Jugendlichen und vor allem Langzeitarbeitslosen, hat sich innerhalb von wenigen Jahren etabliert. Er bedeutet die Fortsetzung der Auseinandersetzungen um die "Soziale Frage" unter veränderten historischen Bedingungen.

In Deutschland wurde in den achtziger Jahren zunächst der Begriff der "neuen Armut" geprägt, der sich bis heute meistens als Reaktion auf die Wahrnehmung einer mangelnden politischen Auseinandersetzung mit dem Phänomen der Armut in der Bundesrepublik versteht. Aber vom Beginn dieser Debatte an war die soziale Ausgrenzung die historisch neue Problemdimension (Lutz 1995). Das Problem stellte sich in den frühen achtziger Jahren ganz konkret durch die Ausgrenzung aufgrund von Reduzierungen der Arbeitslosenunterstützung (Balsen et al. 1983), erweiterte dann aber sehr schnell seine Bedeutung, weil in zahlreichen Untersuchungen auf immer neue Notlagen vieler Menschen im bundesrepulikanischen Alltag hingewiesen wurde.

Dies verstärkte sich noch einmal erheblich mit der Vereinigung, weil nun das Wohlstandsgefälle von West nach Ost dem Problem zusätzliche Brisanz verlieh. Hier überschneidet sich das Thema "Social Exclusion" mit dem der "Inequality" - im Deutschen vielleicht am besten übersetzt mit dem Problem der Gerechtig- keit, auch wenn es durch die ökonomische Ausrichtung in der angelsächsischen Diskussion primär um Einkommensgerechtigkeit geht. Sozialpolitisch stellt es sich als Problem zu großer Einkommensunterschiede, so daß gerade im Zusammenhang mit Informationsgesellschaft von der Gefahr einer "Zweidrittelgesellschaft" gesprochen wird.

Der aktuelle Unterschied zwischen Kontinentaleuropa und den angelsächsischen Ländern wird derzeit vor allem darin gesehen, daß letztere weniger unter dem Problem der Massenarbeitslosigkeit zu leiden haben, daß dafür aber die Schere zwischen den Einkommen zum gravierenden sozialpolitischen Problem geworden ist (Sennett, a.a.O.: 68). Es könnte sein, daß es durch die Vereinigung ein deutsches Spezifikum ist, daß sich beide Probleme gleichermaßen zu einer großen politischen Herausforderung aufschaukeln.

\section{Literatur}

Balsen, Werner; Nakielski, Hans; Roessel, Karl; Winkel, Rolf: Die neue Armut. Ausgrenzung von Arbeitslosen aus der Arbeitslosenunterstützung. Hans-Böckler-Stiftung; Bd. 04, Düsseldorf: Rhein. Journalistenbüro, 1983.

Behrens, Martin; Hardwig, Thomas; Kädtler, Jürgen: Der Industriemeister und der Strukturwandel in der Industrie. Neue Variationen zu einem alten Thema. SOFI-Mitteilungen: Soziologisches Forschungsinstitut Göttingen; Nr. 23; S. 25-44, 1996.

Beicht, Ursula; Berger, Klaus; Herget, Hermann; Krekel, Elisabeth: Berufsperspektiven mit Lehre Wert und Zukunft dualer Berufsausbildung - Beiträge aus Berufsbildungswissenschaft und -praxis. Bielefeld: Bertelsmann 1997.

Czada, Roland: Vereinigungskrise und Standortdebatte. Der Beitrag der Wiedervereinigung zur Krise des westdeutschen Modells. Leviathan: Zeitschrift für Sozialwissenschaft 26(1998)1, 24-59.

Döring, Diether; Nullmeier, Frank; Pioch, Roswitha; Vobruba, Georg: Gerechtigkeit im Wohlfahrtsstaat. REIHE: Standortdebatte. Marburg: SP-Verl. Schüren; 166 S. 1995.

Jagoda, Bernhard: Standort Deutschland im internationalen Wettbewerb. Arbeit und Beruf, Fachzeitschrift für die Aufgaben der Bundesanstalt für Arbeit, 47(1996)3, 65-67.

Kaufmann, Franz-Xaver: Normative Konflikte in Deutschland. Basiskonsens, Wertewandel und soziale Bewegungen. In: Peter L. Berger (Hrsg.): Die Grenzen der Gemeinschaft: Konflikt und Vermitt- 
lung in pluralistischen Gesellschaften. Ein Bericht der Bertelsmann-Stiftung an den Club of Rome; Gütersloh; Verl. Bertelsmann Stiftung; S. 155-197, 1997.

Kroker, Rolf: Deutschland - angeschlagene Standortqualität. WSI-Mitteilungen: Zeitschrift des Wirtschafts- und Sozialwissenschaftlichen Instituts des Deutschen Gewerkschaftsbundes 48(1995)11, 705711.

Lutz, Ronald: Die "Vergessenen der Wende". Kultur der Armut oder Armut der Kultur? Neue Praxis: Zeitschrift für Sozialarbeit, Sozialpädagogik und Sozialpolitik; H. 4; Jg. 25; S. 391-406, 1995.

Müller-Jentsch, Walther: Mitbestimmung als kollektiver Lernprozess. Versuch über die Betriebsverfassung. In: Karsten Rudolph, Christl Wickert (Hg.): Geschichte als Möglichkeit: über die Chancen von Demokratie. Festschrift für Helga Grebing. Essen: Klartext-Verl. 1995.

Sennett, Richard: Der flexible Mensch. Die Kultur des neuen Kapitalismus. Berlin: Berlin Verlag 1998.

SOFI-Soziologisches Forschungsinstitut Göttingen (Hrsg.): Im Zeichen des Umbruchs. Beiträge zu einer anderen Standortdebatte. Opladen: Leske u. Budrich 1995.

Wortmann, Michael; Dörrenbächer, Christoph: Multinationale Konzerne und der Standort Deutschland. Jahrbuch Arbeit und Technik; Jg. 1997; S.2843. 